\title{
Two-Photon 3D Optical Data Storage via Aggregate Switching of Excimer-Forming Dyes
}

\author{
Joseph Lott, Christopher Ryan, Brent Valle, Jack R. Johnson III, David A. Schiraldi, \\ Jie Shan, Kenneth D. Singer,* and Christoph Weder**
}

Current optical data storage (ODS) technologies use onephoton-absorption processes to write data by locally changing the optical properties of the medium. ${ }^{[1,2]}$ Since the lateral dimensions of spots that can be written are near the diffraction limit, significant capacity increases require new approaches such as storage in three dimensions. DVDs, which comprise up to four individually addressable storage layers, exemplify the potential of this concept, but the complexity of producing and using multilayer systems increases with the number of layers. In bulk materials, changes can be confined in the third dimension via nonlinear optical processes, such as two-photon absorption (TPA). ${ }^{[3-6]}$ We have developed a novel ODS system that relies on the optically-induced switching of the aggregation state and fluorescence of a TPA dye in a polymer matrix. Welldefined, $\sim 3 \times 3 \times 6 \mu \mathrm{m}$-large voxels were written with single focused laser pulses and read by confocal laser scanning microscopy. Such ODS systems are easily produced and promise a storage capacity of up to several Tbytes on a DVD-size disk, which is $\sim 100 \times$ higher than that of current commercial ODS technologies. ${ }^{[6,7]}$

The optical changes considered for rewritable and write-once read-many three-dimensional (3D) ODS storage media based on TPA include reversible and irreversible photochemical reactions such as photoisomerizations, ${ }^{[8-10]}$ photo-induced dimerizations, ${ }^{[11,12]}$ photodecompositions, ${ }^{[13,14]}$ and photopolymerizations. ${ }^{[15-17]}$ Fluorescent photochromic systems have attracted particular interest, because the exploitable photophysical processes are fast, efficient, and reversible. ${ }^{[18-20]}$ However, it has been challenging to create fluorescent photochromic materials, which combine high stability, high fluorescence

J. Lott, ${ }^{[+]}$J. R. Johnson III, Prof. D. A. Schiraldi, Prof. C. Weder Department of Macromolecular Science and Engineering

Case Western Reserve University

Cleveland, $\mathrm{OH}, 44106$, USA

E-mail: christoph.weder@unifr.ch

C. Ryan, ${ }^{[+]}$B. Valle, ${ }^{[+]}$Prof. J. Shan, Prof. K. D. Singer,

Department of Physics

Case Western Reserve University Cleveland

$\mathrm{OH}, 44106$, USA

E-mail: kenneth.singer@case.edu

Prof. C. Weder

Adolphe Merkle Institute

Polymer Chemistry and Materials

University of Fribourg

Rte de l'Ancienne Papeterie, CH-1723 Marly 1, Switzerland

[+] J.L., C.R., and B.V. contributed equally to the present work. quantum yield, and large TPA cross-section. We here demonstrate a novel approach to 3D ODS materials, which relies on the switching of the aggregation state of an excimer-forming fluorescent dye with an appreciable TPA cross-section in an inert host polymer.

We have previously reported a range of materials, which change their fluorescence and/or absorption properties upon exposure to heat, ${ }^{[21-23]}$ chemicals, ${ }^{[24]}$ or mechanical forces, ${ }^{[25-27]}$ on account of reversible or irreversible stimulusinduced changes of the aggregation state of the dye molecules. We surmised that such changes could be induced in small volumes by TPA-induced local heating and therefore explored a melt-processed blend of poly(ethylene terephthalate glycol) (PETG) and $1.1 \% \mathrm{w} / \mathrm{w}$ of 1,4 -bis $(\alpha$-cyano-4-octadecyloxystyryl)2,5-dimethoxybenzene ${ }^{[22,23]}$ (C18-RG, Figure 1a) as TPAaddressable ODS medium. C18-RG was selected on account of its significant changes in absorption and emission spectra upon aggregation/dissociation, its high thermal and photochemical stability, and, as demonstrated here, its appreciable TPA cross-section. PETG was chosen as the matrix due to its glassy nature and transparency in the relevant optical window. Its glass transition temperature $\left(T_{\mathrm{g}}\right)$ of $78{ }^{\circ} \mathrm{C}$, which defines the write/erase temperature (vide infra) is sufficiently above ambient temperature and provides excellent stability of the storage medium as discussed below. The solubility phase diagram and aggregation kinetics of C18-RG/PETG blends and similar materials have been previously investigated (Figure 1b). ${ }^{[22,23]}$ Figures $1 \mathrm{~b}$ and 2 illustrate the aggregation state and fluorescence properties of the $1.1 \% \mathrm{w} / \mathrm{w}$ C18-RG/ PETG blend as a function of thermal history. The corresponding normalized absorption and fluorescence spectra are shown in Figure 3. The temperature at which $1.1 \% \mathrm{w} / \mathrm{w}$ of C18-RG is thermodynamically soluble in PETG is $\sim 130^{\circ} \mathrm{C}$ (Figure 1b); note that the dissolution temperature varies with the dye content. Thermodynamically unstable, but kinetically trapped molecular mixtures of the dye and the polymer can be produced by quenching a thermodynamically miscible, melted mixture of the two components $\left(230{ }^{\circ} \mathrm{C}\right.$ for a blend comprising $1.1 \% \mathrm{w} / \mathrm{w}$ dye) to below $T_{\mathrm{g}}$. In this state, the blend film appears yellow (peak wavelength of the absorption spectrum $\lambda_{\text {max }}^{\text {abs }}=447 \mathrm{~nm}$, Figure $3 \mathrm{a}$ ) and displays the green fluorescence (peak wavelength of the fluorescence spectrum $\lambda_{\max }^{\mathrm{fl}}=508 \mathrm{~nm}$, Figure 2a, 3b) that is characteristic of a molecularly mixed blend. Subjecting the quenched blend to temperatures above $T_{\mathrm{g}}$ but below the dissolution temperature leads to stable and pronounced changes in absorption (orange appearance, $\lambda_{\max }^{\mathrm{abs}}=387 \mathrm{~nm}$, Figure $3 \mathrm{a}$ ) and fluorescence (orange, $\lambda_{\max }^{\mathrm{fl}}=$ $542 \mathrm{~nm})$, due to aggregation of the dye molecules. These 
a)

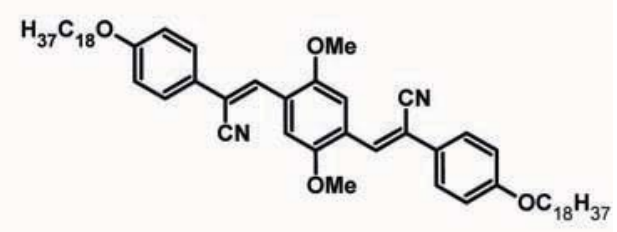

b)

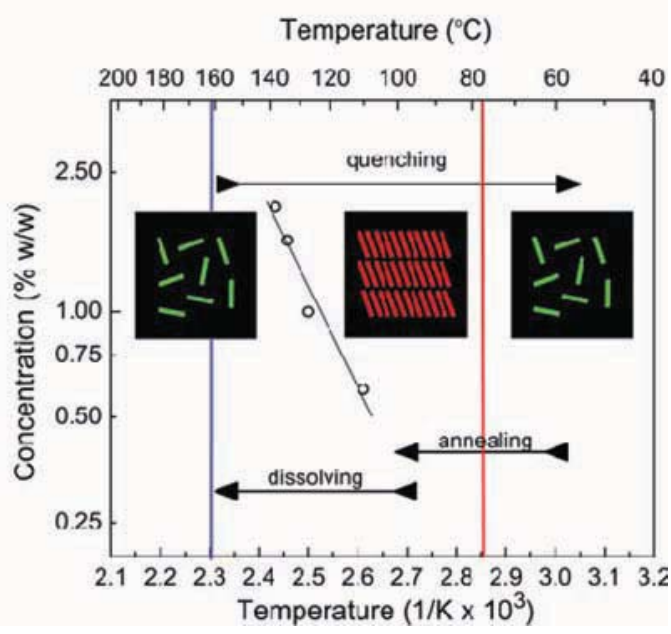

Figure 1. a) Chemical structure of $\mathrm{Cl} 8-\mathrm{RG}$, the dye employed in this study. b) Plot of the (inverse) dissolution temperature of C18-RG/PETC blends as function of dye content. The vertical lines represent the glass transition temperature (red, $78^{\circ} \mathrm{C}$ ) below which the dye is 'immobilized', and the temperature of $160^{\circ} \mathrm{C}$, where blends of all compositions form molecularly mixed melts. Arrows represent the thermal pathways involved in quenching, annealing, and melting. The cartoons depict the dye aggregation state in each temperature regime.

changes are retained if the blend is cooled back to ambient temperature (Figures 2b, 3b). The original state can be restored by subsequently heating the phase-separated blend to above the dissolution temperature (all erase experiments reported here were carried out at $160^{\circ} \mathrm{C}$ ) (Figures 1b, 2c, 3). Thus, the blend employed here can be used as an optically readable storage medium, in which local exposure to welldefined temperatures allows one to write and erase information in two dimensions. In principle, two different modes of operation are possible. Data can be written into a quenched blend (in which the dye molecules are dissolved) by heating to

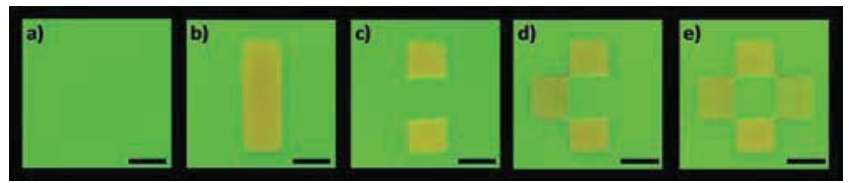

Figure 2. Photographs of a $1.1 \% \mathrm{w} / \mathrm{w}$ C18-RG/PETG blend film demonstrating thermally introduced aggregation (write, $125^{\circ} \mathrm{C}$ ) and dispersion (erase, $160^{\circ} \mathrm{C}$ ) of the dye. a) After compression-molding at $230^{\circ} \mathrm{C}$ and quenching by immersion in ice water. b) After heating a portion of the sample (orange rectangle) through contact with a rectangular copper block $\left(9.0 \times 3.0 \mathrm{~mm}, 125{ }^{\circ} \mathrm{C}, 2.5 \mathrm{~min}\right)$ and subsequent quenching. c) After heating a portion of the sample with the same copper block (turned by $90^{\circ}, 160^{\circ} \mathrm{C}, 30 \mathrm{~s}$ ) and subsequent quenching. d,e) After heating portions of the sample (new orange squares) with a quadratic copper block $\left(3.0 \times 3.0 \mathrm{~mm}, 125^{\circ} \mathrm{C}, 2.5 \mathrm{~min}\right)$ and subsequent quenching. All scale bars represent $3 \mathrm{~mm}$.
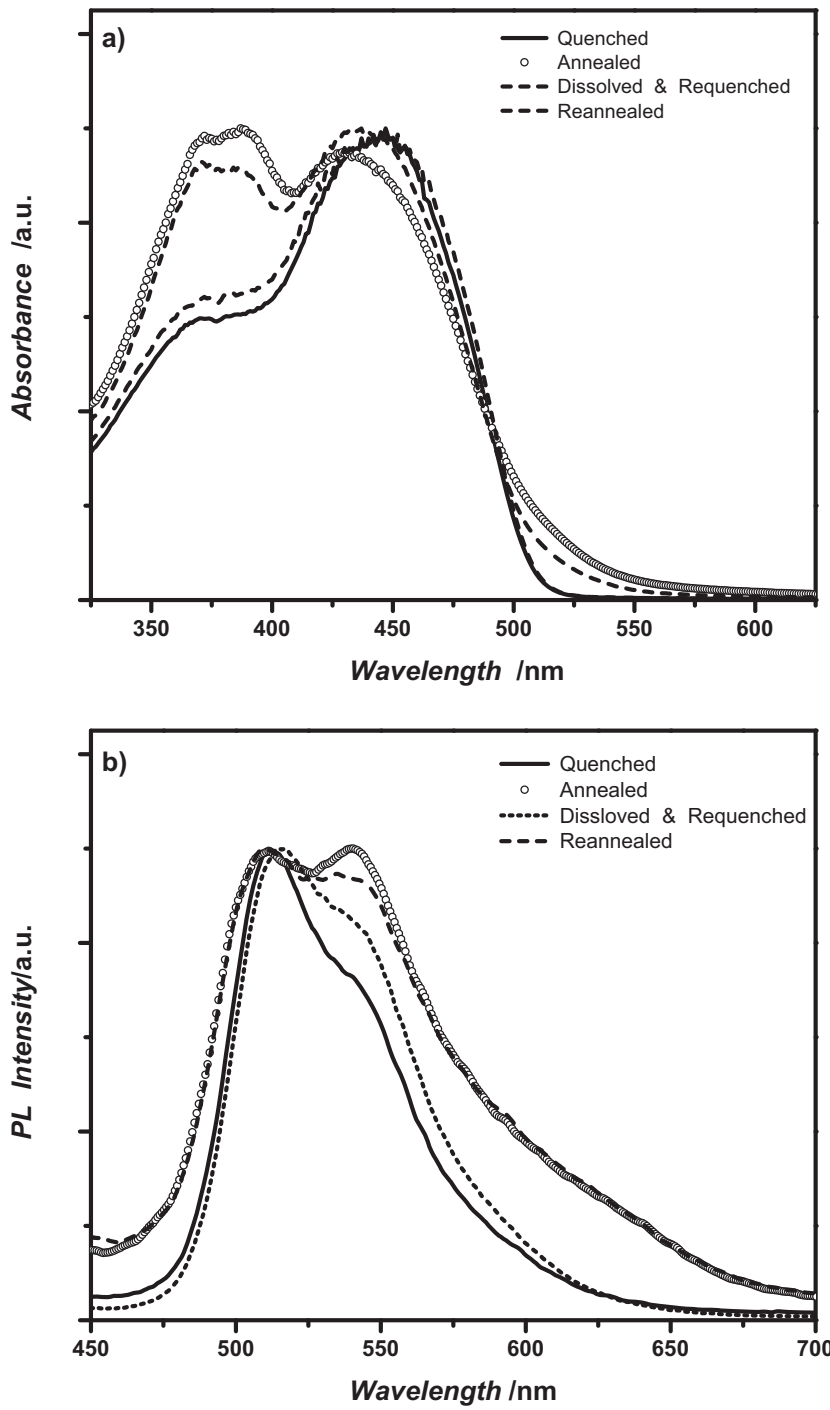

Figure 3. Normalized optical absorption (a) and fluorescence (b) spectra of the sample shown in Figure 2. a) The absorption spectra show the appearance of a band around $387 \mathrm{~nm}$ upon annealing an originally quenched sample at $125{ }^{\circ} \mathrm{C}$, due to aggregation of the dye. The band disappears if the sample is heated to $160^{\circ} \mathrm{C}$ and re-quenched, due to dissolution of the dye. b) Similarly, an emission band around $542 \mathrm{~nm}$ appears and disappears under these conditions.

a temperature above $T_{\mathrm{g}}$ but below the dissolution temperature, and erased through heating above the dissolution temperature. Alternatively, data is written into a phase-separated material by heating above the dissolution temperature and erased by annealing above $T_{\mathrm{g}}$. The cycles illustrated in Figures 2 and 3 show that either starting point is a viable option and that, in principle, many write/read/erase cycles are possible.

The choice to utilize C18-RG for the present optical data storage systems was based on the expectation that this dye, like other cyano-substituted oligo(phenylene vinylene) $\mathbf{s}^{[28-30]}$ possesses an appreciable TPA cross-section, so that the above-described write/erase schemes could be achieved by TPA-induced local heating. The TPA cross-section of C18-RG was measured using the open-aperture Z-scan method 


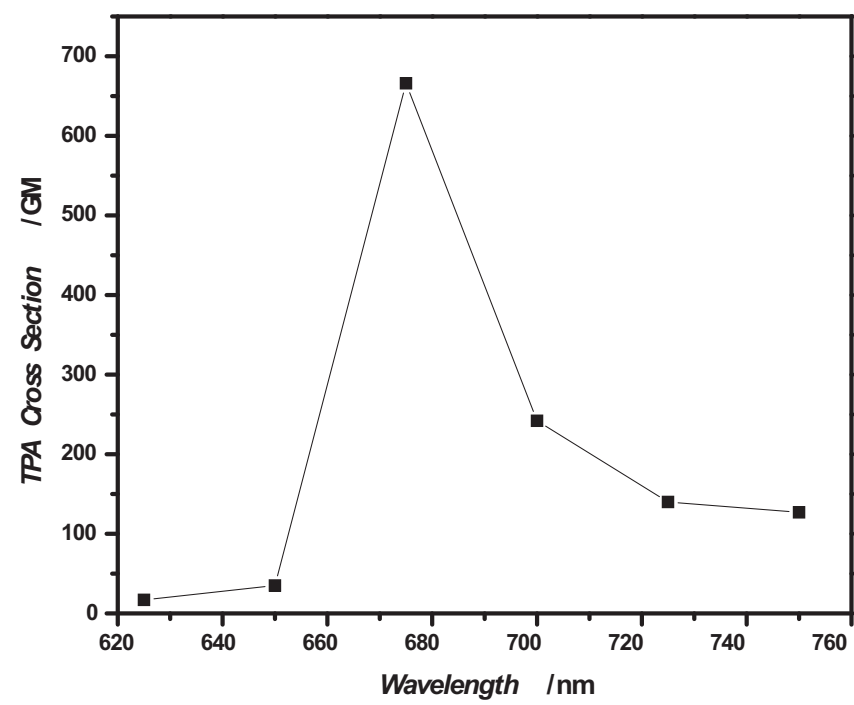

Figure 4. TPA cross-section of C18-RG as a function of wavelength measured by an open-aperture Z-scan measurement $\left(1 \mathrm{GM}=10^{-50} \mathrm{~cm}^{4} \mathrm{~s}^{-1}\right.$ photon $^{-1}$ ).

(Supporting Information, Figure S1). ${ }^{[31,32]}$ Figure 4 shows the TPA cross-section of C18-RG as a function of wavelength in the range of 625 to $725 \mathrm{~nm}$, where the linear absorption is negligible (Figure 3a). The TPA cross-section of the dye varies between 0 and $650 \mathrm{GM}$ with a maximum at $675 \mathrm{~nm}$. The nonlinear absorption is thus significant and comparable to that of similar molecules reported in the literature. ${ }^{[28-30]}$

For TPA-based writing experiments, $1.1 \% \mathrm{w} / \mathrm{w}$ C18-RG/ PETG blend films of a thickness of $150 \mu \mathrm{m}$ were annealed at $90{ }^{\circ} \mathrm{C}$ for 2 days to ensure complete aggregation of the chromophores. Data writing was accomplished by using a Nd:YAG laser in combination with an optical parametric oscillator (OPO), which produced light pulses with a center wavelength of $675 \mathrm{~nm}$, duration of $10 \mathrm{~ns}$, and maximum output energy of $3 \mathrm{~mJ}$. The pulse-to-pulse energy stability was $\sim 20 \%$. These pulses were attenuated and focused onto the storage medium through an oil-immersed objective lens with a numerical aperture of 0.85 . The resulting Gaussian beam was measured to have a waist of $3 \mu \mathrm{m}$ and Rayleigh range of $10 \mu \mathrm{m}$. Each data spot was written by exposing the samples to a single laser pulse. The samples were positioned in $3 \mathrm{D}$ by a computercontrolled 3-axis translation stage. In initial experiments, the average pulse energy was systematically varied. For each trial, a set of spots was written while the average pulse energy was held constant. At pulse energies above $100 \mathrm{~nJ}$ permanent localized damage was observed, while pulse energies below $25 \mathrm{~nJ}$ brought about no visible optical changes. The energy range of 50-65 nJ was found to afford the desired changes. An average pulse energy of $55 \mathrm{~nJ}$ was used for the data writing experiments presented below.

Confocal laser scanning microscopy was used to characterize the voxels written into the C18-RG/PETG blend films. A continuous-wave laser operating at a wavelength of $400 \mathrm{~nm}$ was used to excite the samples and the fluorescence was recorded in two channels corresponding to the integrated intensity in the spectral ranges of 500-525 $\mathrm{nm}$ and $650-800 \mathrm{~nm}$, respectively. These spectral windows were chosen to monitor the

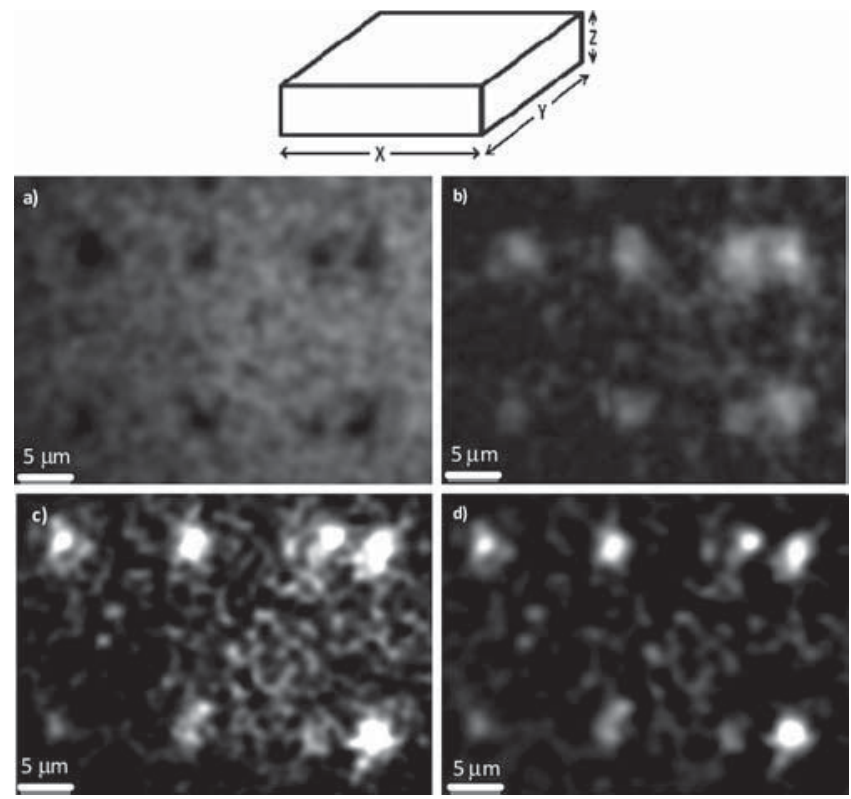

Figure 5. Confocal scanning fluorescence microscopy images of a photopatterned $1.1 \% \mathrm{w} / \mathrm{w} \mathrm{C} 18-\mathrm{RG} / \mathrm{PETG}$ blend film acquired in the plane of the film (XY plane). a) Raw image recorded by the channel monitoring fluorescence in the range of 650-800 nm. b) Raw image recorded by the channel monitoring fluorescence in the range of 500-525 nm. c) Composite image corresponding to the ratio of the intensities of the two channels. d) As in c) but after applying low pass filtering.

aggregated and dispersed state of the chromophores. Confocal microscopy images of a representative sample show eight written data spots at $\sim 10 \mu \mathrm{m}$ below the surface of the film in Figures $\mathbf{5}$ and $\mathbf{6}$. The variations in these data spots were caused by the pulse-to-pulse energy variation of the output of the OPO. Figure 5a shows the intensity image of the sample in a plane parallel to the film surface (XY plane) for fluorescence in the spectral range of $650-800 \mathrm{~nm}$. Orange excimer emission of aggregated dye molecules is observed across the entire sample, except for the written spots, which appear as darker areas, indicative of dispersion of the dye aggregates due to the TPAinduced local heating. The result is further confirmed by the corresponding fluorescence intensity image recorded for the
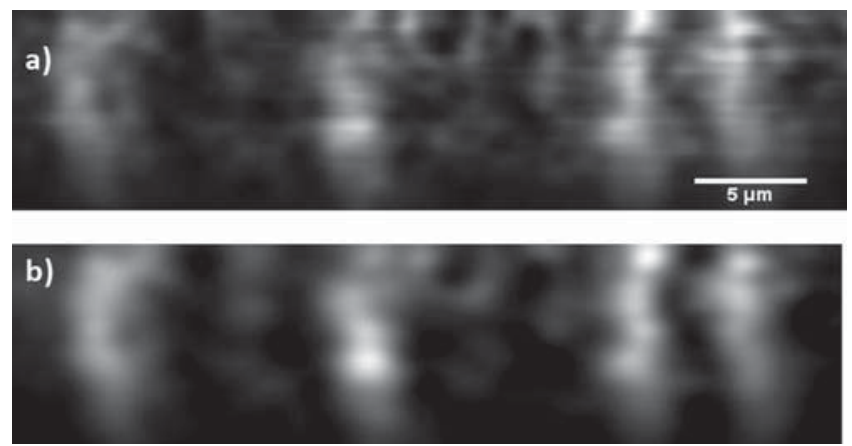

Figure 6. Confocal scanning fluorescence microscopy images of the sample shown in Figure 5 acquired normal to the film surface (ZX plane). Vertical is the z-axis. a) Composite image as computed in Figure $5 c$; b) Cross-section of the composite image after the application of a low pass filter. 
spectral range of 500-525 $\mathrm{nm}$ (Figure 5b). The image shows a complementary behavior; the written spots appear bright, reflecting an increase of the green emission in these areas. No appreciable photo-induced degradation of the films upon writing was observed. The ratio of the emission intensities in the above short and long wavelength windows has been shown to represent a good measure of the aggregation state. ${ }^{[22,23]}$ Thus, it was used to generate a composite image (Figure 5c), which indeed shows a significantly improved contrast. The image contrast can be further enhanced by applying a low pass intensity filter (Figure 5d), which eliminated the effect of the dye aggregates from the image. Details are shown in intensity profiles (Supporting Information, Figure S2) taken along a line passing through the upper four spots in Figures 5a-5d. Further processing involving converting the signal to binary format by applying a threshold value could in principle further enhance the contrast. As is evident from Figure 5, the spot sizes written with the setup employed here have a diameter of $\sim 3 \mu \mathrm{m}$ in the $X Y$ plane. Figure 6 shows the emission intensity profile of the top four spots shown in Figure $5 \mathrm{c}$ as recorded in the ZX plane. The dimensions of the data spots in the ZX direction $(\sim 6 \mu \mathrm{m})$ are a bit larger than those in the XY-plane $(\sim 3 \mu \mathrm{m})$, which are mostly limited by the dimensions of the beam focus. The current results reveal clearly that the TPA-based writing process allows one to write voxels that are microscopically localized in all 3Ds. Because the writing process requires a threshold temperature, it should be possible to achieve a data spot substantially smaller than the writing beam size. Below the polymer's $T_{\mathrm{g}}$, the translational mobility of the dye molecules is negligible ${ }^{[22,23]}$ and the morphology of the dye/host systems should be stable for years. Erasing data is possible by heating above $T_{\mathrm{g}}$ and re-aggregate the chromophores, ${ }^{[23]}$ as has been illustrated in Figure 2, either by direct exposure to heat or TPA-induced heating.

In summary, we have demonstrated a new ODS system that relies on the optically-initiated, thermally induced switching of the aggregation state of an excimer-forming, fluorescent TPA dye in a polymer matrix. Such blends can be easily, inexpensively and rapidly fabricated in large quantities using simple meltprocessing techniques. Well-defined voxels with dimensions of $\sim 3 \times 3 \times 6 \mu \mathrm{m}$ have been written through the exposure of the blend to single laser pulses. The voxel size is comparable to the focal point volume of the writing laser, suggesting that thermal transport does not place a lower limit to the voxel size, at least not at the length scales used here. A diffraction limited laser beam can be achieved by optimizing the optical setup. In that case the volume for efficient TPA is limited to a space slightly smaller than $0.4 \mu \mathrm{m}$ in each dimension given the wavelength and numerical aperture used. Such a scheme can be used to potentially write several terabytes of binary data in a disk of size of common CDs and DVDs.

\section{Experimental Section}

Materials: The chromophore 1,4-bis ( $\alpha$-cyano-4-octadecyloxystyryl)-2,5dimethoxybenzene (C18-RG) was synthesized as previously described. ${ }^{23]}$ Poly(ethylene terephthalate glycol) (PETG) Eastar 6763 and spectroscopic grade toluene were obtained from Eastman Chemical Company and Burdick \& Jackson and were used as received.
Sample Preparation: A blend of C18-RG and PETG (nominal dye content $2 \% \mathrm{w} / \mathrm{w}$ ) was prepared using a Haake Rheocord 9000 batch mixer at $230{ }^{\circ} \mathrm{C}$ for $5 \mathrm{~min}$. This blend was compression-molded between Kapton sheets into thin films of a thickness of $\sim 150 \mu \mathrm{m}$ in a Carver melt press at $230{ }^{\circ} \mathrm{C}$ and 4 tons for $3 \mathrm{~min}$. Aluminum spacers were used to control the film thickness. Upon removal from the press the films were quenched by immediate immersion into ice water. Some of the films thus produced were annealed for 2 days at $90^{\circ} \mathrm{C}$ under an $\mathrm{N}_{2}$ (g) atmosphere.

UV-vis Spectroscopy: UV-vis absorption spectra were recorded on a Perkin Elmer Lambda 800 spectrophotometer and used to determine the actual dye concentration in the blend $(1.1 \% \mathrm{w} / \mathrm{w})$ based on the film thickness and the extinction coefficient of C18-RG $\left(31250 \mathrm{~L} \cdot \mathrm{mol}^{-1} \cdot \mathrm{cm}^{-1}\right)$.

Photoluminescence Spectroscopy: Photoluminescence (PL) spectra were recorded using a Photon Technology International QuantaMaster 40 spectrophotometer under excitation at $423.5 \mathrm{~nm}$; the spectra were corrected for the instrument throughput and the detector response.

Z-Scan Measurements: The TPA cross-section of C18-RG was measured at various wavelengths using the open-aperture Z-scan technique. ${ }^{[31,32]}$ A Ti:Sapphire regenerative amplifier (CPA-2010, from Clark-MXR) with $200 \mathrm{fs}$ pulse duration and $1 \mathrm{~mJ}$ pulse energy was used to pump a traveling-wave optical parametric amplifier of superfluorescence (TOPAS, Light Conversion Ltd.). The output from the TOPAS was passed through a spatial filter to isolate the lowest-order transverse mode of the beam. The Z-scan setup included a reference channel to minimize shot-to-shot noise and to ignore pulses deviating more than two standard deviations from the mean. Beam waists were measured using a rotating slit beam profiler (BP104-VIS, Thorlabs Inc.). Data was acquired from the photodiodes in combination with a gated boxcar integrator (SR200 series, Stanford Research Systems) while the sample was translated through the focus of a $20 \mathrm{~cm}$ focal length lens by a computer-controlled translation stage. The accuracy of the Z-scan measurement was probed by measuring the TPA cross-section of Rhodamine 6G $\left(2.13 \cdot 10^{-3} \mathrm{M}\right.$ in methanol) at a wavelength of $685 \mathrm{~nm}$ and the measured value $(76.9 \mathrm{GM})$ is consistent with values in the literature ( 55-97 GM). ${ }^{[33]}$ The TPA cross-section of C18-RG was subsequently measured in a fused quartz cuvette ( $1 \mathrm{~mm}$ path-length) at wavelengths between 625 and $725 \mathrm{~nm}$.

3D Data Storage: Patterns were written by translating the C18-RG/ PETC blend films through a focused writing beam. A Nd:YAG laser (Surelite II-10, Continuum) was used to pump an optical parametric oscillator (Surelite OPO, Continuum) to produce light pulses of wavelength of $675 \mathrm{~nm}$, pulse duration of $10 \mathrm{~ns}$, and pulse energy of $3 \mathrm{ml}$. These pulses were then attenuated, and focused onto the sample films by an oil-immersed objective lens with a numerical aperture of 0.85 . Films were placed on a computer controlled 3-axis translation stage. Data was written into the film by single laser pulses as the film was translated. This was achieved through simultaneous computer control of the Q-switch of the Nd:YAG pump laser and the position of the film. The pulse energy was varied between 1 and $1000 \mathrm{n}$ ) by inserting neutral density filters into the beam path. Energy measurements were made at the sample position with a calibrated silicon diode to determine the amount of energy delivered to the sample.

Data Read-Out: The written data was read with a Fluoview 1000 confocal scanning microscope operated in fluorescence detection mode using an excitation wavelength of $400 \mathrm{~nm}$. For the results reported here an objective $(100 \times, 1.0 \mathrm{NA})$ was used to provide a spatial resolution of $\sim 250 \mathrm{~nm}$. The fluorescence from the sample was passed through a set of dichroic mirrors and band pass filters and fluorescence in the range of $500-525 \mathrm{~nm}$ and in the range of $650-800 \mathrm{~nm}$ was detected by two photomultiplier tubes. 


\section{Acknowledgements}

This material is based upon work supported by the (NSF DMR-0423914, Center for Layered Polymer Systems at Case Western Reserve University). C.W. acknowledges support from the Adolphe Merkle Foundation.

[1] M. Wuttig, N. Yamada, Nat. Mater. 2007, 6, 824.

[2] H. Mustroph, M. Stollenwerk, V. Bressau, Angew. Chem. Int. Ed. 2006, 45, 2016.

[3] D. A. Parthenopoulos, P. M. Rentzepis, Science 1989, 245, 843.

[4] D. Psaltis, Science 2002, 298, 1359.

[5] R. P. C. Won, Nat. Photonics published online: 16 November 2006, DOI:10.1038/nphoton.2006.47.

[6] M. Pawlicki, H. A. Collins, R. G. Denning, H. L. Anderson, Angew. Chem. Int. Ed. 2009, 48, 3244.

[7] E. Walker, P. M. Rentzepis, Nat. Photonics 2008, 2, 406.

[8] F. Gallego-Gomez, F. Del Monte, K. Meerholz, Nat. Mater. 2008, 7, 490.

[9] C. J. Carling, J. C. Boyer, N. R. Branda, J. Am. Chem. Soc. 2009, 131, 10838.

[10] D. Gindre, A. Boeglin, A. Fort, K. D. Mager, L. Dorkenoo, Opt. Express 2006, 14, 9896.

[11] K. Iliopoulos, O. Krupka, D. Gindre, M. Salle, J. Am. Chem. Soc. 2010, 132, 14343.

[12] B. Lohse, R. Vestberg, M. T. Ivanov, S. Hvilsted, R. H. Berg, C. J. Hawker, P. S. Ramanujam, Chem. Mater. 2008, 20, 6715.

[13] H. A. Al Attar, O. Taqatqa, J. Opt. A, Pure Appl. Opt. 2003, 5, S487.

[14] M. Sakamoto, T. Tachikawa, M. Fujitsuka, T. Majima, Adv. Mater. 2008, 20, 3427.
[15] A. Khan, A. E. Daugaard, A. Bayles, S. Koga, Y. Miki, K. Sato, J. Enda, S. Hvilsted, G. D. Stucky, C. J. Hawker, Chem. Comm. 2009, 4, 425.

[16] R. Castagna, F. Vita, D. E. Lucchetta, L. Criante, F. Simoni, Adv. Mater. 2009, 21, 589.

[17] K. Hirabayashi, H. Kanbara, Y. Mori, T. Kurihara, M. Shimizu, T. Hiyama, Appl. Opt. 2007, 46, 8402.

[18] C. C. Corredor, Z. L. Huang, K. D. Belfield, Adv. Mater. 2006, 18, 2910.

[19] S. Kawata, Y. Kawata, Chem. Rev. 2000, 100, 1777.

[20] M. Irie, Chem. Rev. 2000, 100, 1685.

[21] B. R. Crenshaw, C. Weder, Adv. Mater. 2005, 17, 1471.

[22] B. R. Crenshaw, J. Kunzelman, C. E. Sing, C. Ander, C. Weder, Macromol. Chem. Phys. 2007, 208, 572.

[23] M. Kinami, B. R. Crenshaw, C. Weder, Chem. Mater. 2006, 18, 946.

[24] J. Kunzelman, B. R. Crenshaw, C. Weder, J. Mater. Chem. 2007, 17, 2989.

[25] J. Lott, C. Weder, Macromol. Chem. Phys. 2010, 211, 28.

[26] C. Löwe, C. Weder, Synthesis 2002, 9, 1185.

[27] B. R. Crenshaw, C. Weder, Chem. Mater. 2003, 15, 4717.

[28] S. J. Chung, M. Rumi, V. Alain, S. Barlow, J. W. Perry, S. R. Marder, J. Am. Chem. Soc. 2005, 127, 10844.

[29] S. J. K. Pond, O. Tsutsumi, M. Rumi, O. Kwon, E. Zojer, J. Bredas, S. R. Marder, J. W. Perry, J. Am.Chem. Soc. 2004, 126, 9291.

[30] H. H. Fang, Q. D. Chen, J. Yang, H. Xia, B. R. Gao, J. Feng, Y. G. Ma, H. B. Sun, J. Phys. Chem. C 2010, 114, 11958.

[31] M. Sheik-Bahae, A. A. Said, E. W. Van Stryland, Opt. Lett. 1989, 4, 955.

[32] E. W. Van Stryland, M. Sheik-Bahae, "Z-Scan," in Characterization Techniques and Tabulations for Organic Nonlinear Optical Materials (Eds. M. G. Kuzyk, C. W. Dirk), Marcel Dekker, Inc.1998 pp. 655-692.

[33] N. S. Makarov, M. Drobizhev, A. Rebane, Opt. Express 2008, 16, 4029. 